\title{
Aarnoo Prevalência e fatores associados à violência por parceiro íntimo na gestação em Caxias, Maranhão, 2019-2020*
}

doi: 10.1590/51679-49742021000200012

\author{
Prevalence and factors associated with intimate partner violence during pregnancy in Caxias, \\ state of Maranhão, Brazil, 2019-2020
}
Prevalencia y factores asociados con la violencia por la pareja durante el embarazo en Caxias, en el estado de Maranhão, Brasil, 2019-2020

\author{
Hayla Nunes da Conceição' 1 - (1) orcid.org/0000-0001-6035-8280 \\ Sara Ferreira Coelho ${ }^{1}$ - (1) orcid.org/0000-0002-2315-3902 \\ Alberto Pereira Madeiro² - (1) orcid.org/0000-0002-5258-5982 \\ 'Universidade Federal do Piauí, Programa de Pós-Graduação em Saúde e Comunidade, Teresina, PI, Brasil \\ ${ }^{2}$ Universidade Estadual do Piauí, Centro de Ciências da Saúde, Teresina, PI, Brasil
}

\section{Resumo}

Objetivo: Analisar a prevalência e fatores associados à violência por parceiro íntimo na gestação. Métodos: Estudo transversal, com dados obtidos de entrevistas com grávidas de 10 a 49 anos de idade, no terceiro trimestre gestacional, residentes em Caxias, Maranhão, Brasil (2019-2020). Utilizou-se o instrumento World Health Organization Violence Against Women Study para identificação da forma de violência. Realizou-se análise hierarquizada por regressão logística múltipla. Resultados: Foram entrevistadas 233 gestantes. A violência na gestação apresentou prevalência de 33,0\%, com predomínio da violência psicológica (18,9\%). No modelo hierarquizado final, a faixa etária da mulher $<20$ anos $\left(0 \mathrm{Raj}=2,09-\mathrm{IC}_{95 \%}\right.$ $1,17 ; 3,54)$ e o consumo de drogas ilícitas pelo parceiro (0Raj=8,78 - $\left.\mathrm{IC}_{95 \%} 2,13 ; 28,92\right)$ mantiveram-se associados ao desfecho de violência. Conclusão: A violência na gestação apresentou elevada prevalência, sendo a idade jovem da mulher e o uso de substâncias ilícitas pelo parceiro fatores associados a sua ocorrência.

Palavras-chave: Violência contra a Mulher; Violência por Parceiro Íntimo; Gravidez; Maus-Tratos Conjugais; Estudos Transversais.

\footnotetext{
*Artigo derivado da dissertação de mestrado acadêmico intitulada 'Violência por parceiro íntimo na gestação: prevalência e fatores associados em Caxias, Maranhão', apresentada por Hayla Nunes da Conceição junto ao Programa de Pós-Graduação em Saúde e Comunidade da Universidade Federal do Piauí em 2020.
}

Endereço para correspondência:

Hayla Nunes da Conceição - Av. Frei Serafim, no 2280, Centro-Sul, Teresina, PI, Brasil. CEP: 64001-450

E-mail: haylanunes_cx@hotmail.com 


\section{Introdução}

A violência contra a mulher é importante causa de morbimortalidade feminina. Por sua elevada magnitude e ampla disseminação, essa forma de violência representa um desafio para a Saúde Pública de todo 0 mundo. ${ }^{1}$ Dados de inquérito conduzido entre 2010 e 2017, com mulheres na idade dos 15 aos 49 anos, residentes em 46 países, evidenciaram que a violência ocorre principalmente no ambiente familiar e tem como principal agressor o parceiro íntimo. Ademais, 0 abuso físico e/ou a violência sexual em algum momento da vida variam de 3,5 a $46,0 \% .{ }^{2}$ A violência por parceiro íntimo (VPI) associa-se a estresse pós-traumático, uso abusivo de álcool e drogas ilícitas, infecções sexualmente transmissíveis (IST), depressão, ideações suicidas e morte.,

\section{Mulheres jovens, com histórico de violência pessoal ou familiar, com parceiro sem ocupação e/ou usuário de drogas ilícitas, apresentaram maior chance de serem vítimas de violência na gestação.}

Gestantes não estão isentas de VPI. Incluse, os episódios de violência podem iniciar ou aumentar durante 0 ciclo gravídico-puerperal. Embora haja disparidade de prevalências nas várias regiões do mundo, as taxas de violência por parceiro íntimo na gestação (VPIG) são mais elevadas na África e na América Latina. ${ }^{5}$ No Brasil, estudo realizado na cidade de São Paulo e na zona rural de Pernambuco mostrou prevalência de VPIG de $8,0 \%$ e $12,0 \%$, respectivamente, no período de 2000 a $2003 .{ }^{6}$ Outra pesquisa, esta sobre a região Nordeste em 2016, observou que $20,1 \%$ das gestantes sofreram violência física praticada pelo parceiro. ${ }^{7}$ No entanto, a violência psicológica é a mais frequentemente relatada por mulheres expostas à VPIG.,

A VPIG acomete mulheres de todas as classes sociais. ${ }^{3,10}$ Contudo, as mais jovens, com baixas escolaridade e renda, com histórico de vitimização antes da gestação e que presenciaram suas mães serem agredidas pelo parceiro íntimo têm sido apontadas como as mais vulneráveis à violência na gravidez. ${ }^{11,12}$ Início precoce da atividade sexual, ter três ou mais filhos, ${ }^{13}$ referência de múltiplos parceiros sexuais, ${ }^{11}$ gravidez não planejada, ${ }^{7}$ aborto induzido ou tentativa de interromper a gestação ${ }^{8}$ também são relacionados como fatores associados à violência no período gestacional. Além disso, uso de álcool e substâncias ilícitas, baixa escolaridade, condições socioeconômicas desfavoráveis e desemprego do parceiro íntimo determinam maior risco de sofrer VPIG. ${ }^{6,10,12,14}$

São bem conhecidos os desfechos negativos da VPIG para a saúde materna e fetal, como aborto espontâneo, ruptura prematura de membranas, parto prematuro, aumento de complicações no trabalho de parto, maior número de cesáreas e hospitalizações, baixo peso ao nascer, natimortalidade e morte perinatal. ${ }^{6,12,15}$ Fortemente associados ao tipo, à magnitude e à persistência da VPI são também a baixa ou nenhuma adesão às consultas de pré-natal, redução no tempo de amamentação, transtornos mentais, depressão pós-parto e baixa qualidade de vida da mulher.,

Apesar de sua relevância, os dados sobre a magnitude da VPIG ainda são escassos, e a quantidade insuficiente de informações pode ser um obstáculo à prevenção baseada em evidências, impossibilitando a identificação dos grupos de risco., ${ }^{1,2}$ Observa-se que esses dados variam com frequência, a depender do contexto sociocultural em que a mulher se encontra. Dessa forma, torna-se importante conhecer o panorama da VPIG em locais sem informações sobre o tema, para subsidiar o planejamento de estratégias de redução dos casos em nível local, além de sensibilizar os profissionais encarregados da atenção às mulheres gestantes sobre a relevância desse problema de Saúde Pública.

Assim, o estudo objetivou analisar a prevalência e os fatores associados à violência por parceiro íntimo na gestação, no município de Caxias, estado do Maranhão, Brasil.

\section{Métodos}

Trata-se de estudo transversal, com gestantes de Caxias, MA, conduzido entre setembro de 2019 e março de 2020.

Caxias ocupa área de 5.196,769 $\mathrm{km}^{2}$ e é a terceira maior cidade em extensão territorial do Maranhão. Situado na região leste do estado, a $374 \mathrm{~km}$ da capital São Luís, e a $70 \mathrm{~km}$ da capital piauiense, Teresina, 0 município apresenta população estimada de 165.525 habitantes em 2020, em sua maior parte do sexo 
feminino (51,6\%) e sem nenhum grau de instrução ou apenas o ensino fundamental incompleto (79,5\%). 0 município conta com 36 unidades básicas de saúde (UBS), que prestam assistência primária à saúde de $70,4 \%$ de sua população. ${ }^{16}$

As participantes do estudo foram gestantes que se encontravam no terceiro trimestre de gestação (a partir da $28^{\mathrm{a}}$ semana), residentes do município e com acompanhamento pré-natal pelo Sistema Único de Saúde (SUS) no período analisado. Foram excluídas da pesquisa mulheres com idade inferior a 18 anos cujos responsáveis não assinaram o Termo de Consentimento Livre e Esclarecido, embora aceitassem participar, além de grávidas que não apresentavam capacidade para responder à entrevista.

Com base no total de gestantes no terceiro trimestre de gravidez $(\mathrm{n}=327)$, durante o período de estudo, erro de $5 \%$, nível de confiança de $95 \%$, poder de $80 \%$ e acréscimo de $10 \%$ para perdas, foi estimada amostra mínima de 204 mulheres. Utilizou-se amostragem por conveniência, dada a impossibilidade de obtenção de amostra aleatória representativa da população de gestantes de Caxias. Todas as gestantes no terceiro trimestre cadastradas foram contatadas.

A coleta de dados foi realizada por pesquisadora treinada e ocorreu, prioritariamente, nas UBS, em sala reservada, antes ou após as consultas de pré-natal, sem a presença de acompanhantes. Para as gestantes não encontradas nas UBS, a entrevista foi realizada no domicílio, exceto quando estavam presentes outras pessoas no local ou havia impedimento à entrevista em ambiente reservado. Para as entrevistas domiciliares, após três tentativas de visita sem sucesso, a gestante foi excluída.

Os dados foram coletados em entrevistas face a face, utilizando-se dois formulários estruturados com perguntas fechadas.

0 primeiro formulário consistia de um instrumento de investigação da VPI, conhecido como World Health Organization Violence Against Women Study (WHO VAW), validado no Brasil. ${ }^{17} 0 \mathrm{WHO}$ VAW, utilizado para avaliar a ocorrência de violência no período gestacional, apresenta 13 questões divididas em 3 subescalas, que avaliam a ocorrência de violência física, sexual e psicológica, sendo 4 itens para identificar a violência psicológica, 6 a violência física e 3 a violência sexual. 0 formulário WHO VAW considera que uma resposta afirmativa já indica caso de violência. ${ }^{17} 0$ estudo considerou caso de VPIG cada relato, pelas entrevistadas, de algum tipo de violência (física e/ou sexual e/ou psicológica) cometida pelo parceiro íntimo durante a gravidez. Entendeu-se como parceiro íntimo um namorado/ex-namorado, marido/ex-marido ou qualquer indivíduo que, mesmo sem união formal, tenha estabelecido relação afetiva e mantém ou manteve relação sexual com a mulher. ${ }^{18}$

0 segundo formulário abordava as seguintes variáveis:

a) Sociodemográficas

- Faixa etária (em anos: 10-14; 15-19; 20-29; 30 39; 40-49);

- Raça/cor da pele (branca; preta; parda; amarela; indígena);

- Escolaridade (em anos completos de estudo: 0-4; $5-8 ; 9-11 ; \geq 12)$;

- Situação conjugal (com parceiro; sem parceiro)

- Fonte de renda própria (sim; não);

- Situação ocupaçional (com ocupação; sem ocupação);

- Condição de moradia (casa própria; casa alugada).

b) Obstétricas

- Número de gestações $(\leq 2 ;>2)$;

- Número de partos $(\leq 2 ;>2)$;

- Aborto anterior (sim; não);

- Número de consultas de pré-natal $(<6 ; \geq 6)$.

c) Relacionadas à saúde

- Consumo de álcool (sim; não) e de drogas ilícitas (sim; não).

d) Histórico de violência

- Violência psicológica antes (sim; não; não se aplica) e depois dos 15 anos (sim; não; não se aplica);

- Violência física antes (sim; não; não se aplica) e depois dos 15 anos (sim; não; não se aplica);

- Violência sexual antes (sim; não; não se aplica) e depois dos 15 anos (sim; não; não se aplica);

- Agressão materna presenciada (sim; não);

- Tipo de agressão materna presenciada (psicológica; física; sexual).

e) Sobre o parceiro íntimo

- Faixa etária (em anos: 10-14; 15-19; 20-29; 3039; 40-49; $\geq 50$ );

- Raça/cor da pele (branca; preta; parda; amarela; indígena);

- Escolaridade (em anos de estudo: 0-4; 5-8; 9-11; $\geq 12$ ); 
- Ocupação profissional (com ocupação; sem ocupação);

- Consumo de álcool (sim; não) e de drogas ilícitas (sim; não).

Um teste-piloto foi desenvolvido com dez gestantes, para aprimorar o formulário. Após ajustes, os dados coletados no pré-teste foram descartados. No momento da entrevista, as gestantes identificadas como vítimas de violência foram orientadas a procurar os serviços de atendimento especializado de atenção à mulher em situação de violência existentes no município.

Os dados foram analisados por estatística descritiva, com frequências absoluta e relativa, com o uso do programa Statistical Package for the Social Sciences (SPSS) versão 20. A prevalência da violência foi calculada identificando-se a ocorrência isolada, por tipo de violência, e a simultânea, de dois ou mais tipos de violência sofridos por cada participante, utilizando-se o diagrama de Venn. Para a análise inferencial, todas as variáveis independentes foram dicotomizadas.
Empregou-se a regressão logística para avaliar a associação entre as variáveis independentes (características sociodemográficas da gestante e do parceiro íntimo; características obstétricas; comportamentos relacionados à saúde; histórico de violência da mulher) e a variável dependente (VPIG), expressa em valores de odds ratio brutos (ORbr) e ajustados (ORaj), com intervalo de confiança de $95 \%\left(\mathrm{IC}_{95 \%}\right)$. Como ponto de corte para o diagnóstico de multicolinearidade, foi adotado um fator de inflação da variância (VIF) abaixo de 10.

As variáveis independentes foram tratadas de maneira hierarquizada (Figura 1), descrevendo-se as relações entre fatores de risco de VPIG, com três níveis de determinantes: distal, intermediário e proximal. A hierarquização das variáveis tomou como referência estudos anteriores. ${ }^{12,19} 0$ nível distal ao desfecho foi composto por variáveis sociodemográficas da mulher e do parceiro; 0 intermediário, por características obstétricas; e o proximal, por aspectos comportamentais

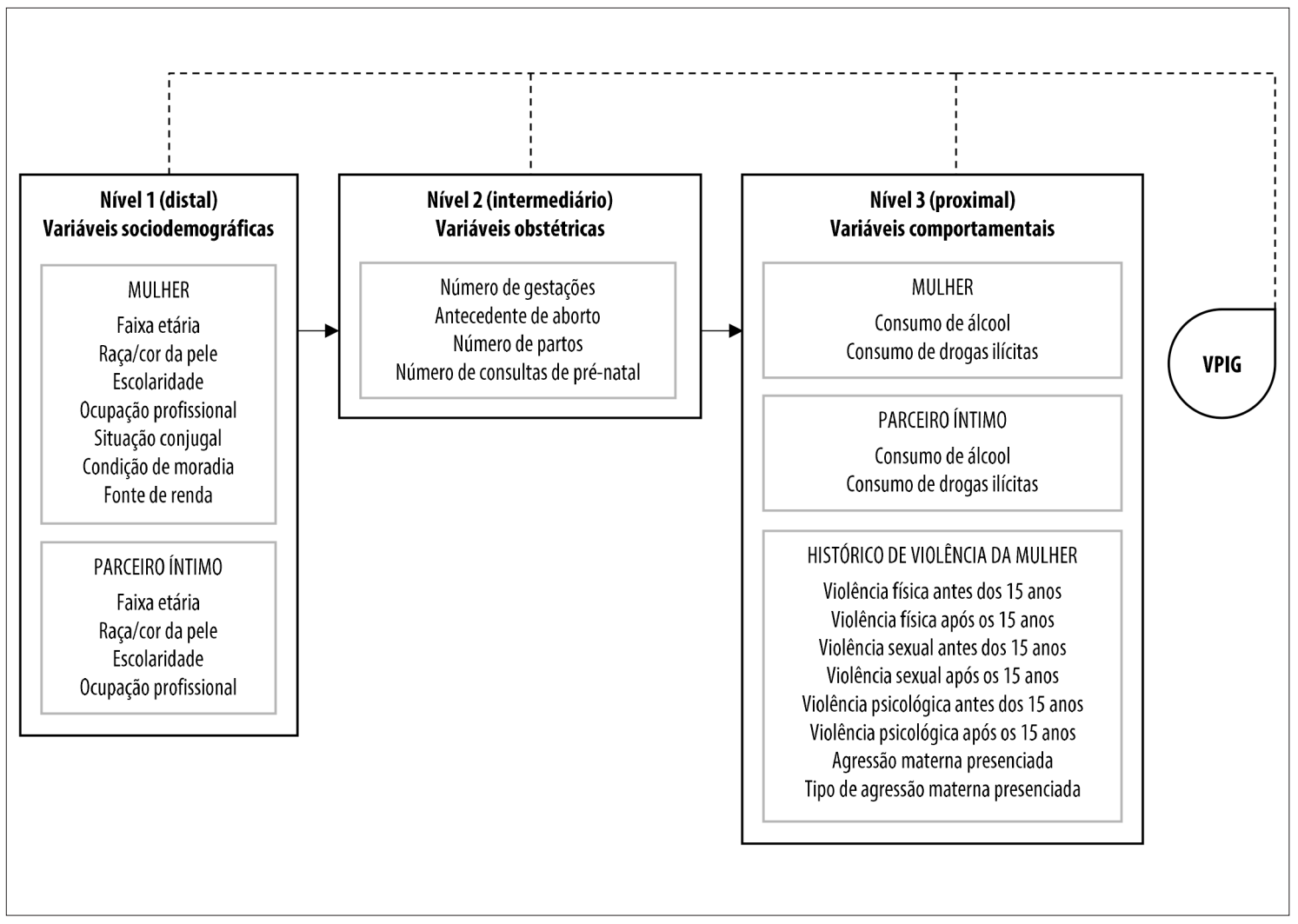

Figura 1 - Modelo hierarquizado dos fatores de risco para a violência por parceiro íntimo na gestação (VPIG) 
da mulher e do parceiro, além de características referentes ao histórico de violência. 0 modelo hierárquico foi analisado seguindo a direção distal-proximal. Inicialmente, foram incluídas no modelo somente as variáveis do nível distal, permanecendo aquelas que apresentaram $p \leq 0,10$. Em seguida, foram incluídas as variáveis do nível intermediário, permanecendo aquelas desse nível com $p \leq 0,10$ e ajustadas para as variáveis do nível anterior. No nível proximal, as variáveis foram ajustadas por todas dos níveis anteriores. No modelo final, foram consideradas associadas ao desfecho as variáveis que apresentaram $p<0,05$.

0 projeto da pesquisa foi aprovado pelo Comitê de Ética em Pesquisa Humana da Universidade Federal do Piauí (CEP/UFPI): Certificado de Apresentação para Apreciação Ética (CAAE) $n^{0}$ 16088619.9.0000.5214/ Parecer $\mathrm{n}^{0} 3.429 .407$, emitido em $1^{\circ}$ de julho de 2019. Todas as gestantes selecionadas, como condição para participar do estudo, assinaram o Termo de Consentimento Livre e Esclarecido.

\section{Resultados}

Foram entrevistadas 233 gestantes e houve nove exclusões. A prevalência de algum tipo de violência na gestação foi de 33,0\% (n=77), sendo a violência psicológica isolada a mais frequente $(18,9 \%)$, seguida pela sobreposição de violência física e psicológica $(5,2 \%)$ e da violência física isolada (4,3\%) (Figura 2). Insultos representaram o tipo de violência psicológica mais praticado pelos parceiros $(n=46 ; 19,7 \%)$, enquanto tapas $(n=18 ; 7,7 \%)$ e empurrões $(n=15 ; 6,4 \%)$, os atos mais comuns de violência física. A prática sexual forçada fisicamente pelo companheiro foi a mais relatada $(n=7 ; 3,0 \%)$ entre os casos de violência sexual.

Houve associação entre VPIG e idade inferior a 20 anos $\left(0 \mathrm{Rbr}=2,59-\mathrm{IC}_{95 \%}\right.$ 1,47;4,59). Gestantes cujos parceiros não tinham ocupação $\left(0 \mathrm{Rbr}=3,32-\mathrm{IC}_{95 \%}\right.$ $1,51 ; 7,02)$ e faziam uso de drogas ilícitas $(0 \mathrm{Rbr}=11,33$ - $\left.\mathrm{IC}_{95 \%} 3,15 ; 40,80\right)$ apresentaram mais chances de serem vítimas de violência durante a gestação (Tabela 1).

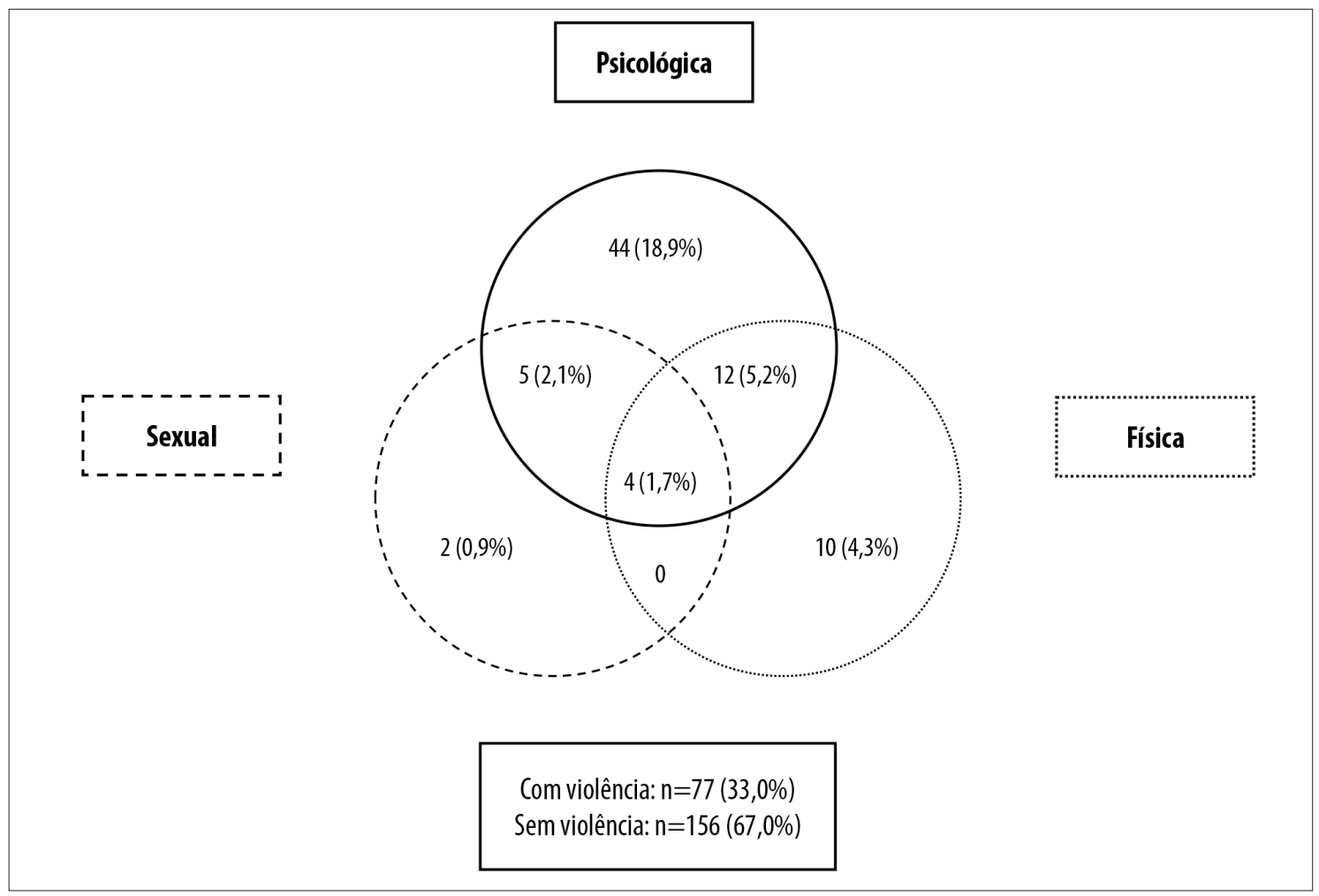

Figura 2 - Prevalência de violência psicológica, física e sexual cometida pelo parceiro íntimo na gestação, Caxias, Maranhão, 2019-2020 
Também se observou maior chance de VPIG entre aquelas que sofreram violência física antes $(0 \mathrm{Rbr}=2,63$ - $\left.\mathrm{IC}_{95 \%} 1,46 ; 4,73\right)$ e depois dos 15 anos (0Rbr $=2,45$ - IC $\left._{95 \%} 1,22 ; 4,93\right)$, violência sexual antes dos 15 anos $\left(0 \mathrm{Rbr}=3,30-\mathrm{IC}_{95 \%} 1,13 ; 9,67\right)$ e violência psicológica antes $\left(0 \mathrm{Rbr}=3,28-\mathrm{IC}_{95 \%}, 1,84 ; 5,89\right)$ e depois dos 15 anos (ORbr $\left.=2,77-\mathrm{IC}_{95 \%} 1,59 ; 4,94\right)$; e também entre as gestantes que presenciaram agressões maternas física e psicológica $\left(0 \mathrm{Rbr}=2,90-\mathrm{IC}_{95 \%}, 1,03 ; 8,14\right)$ (Tabela 2).

A Tabela 3 apresenta os dados da análise hierarquizada. No nível 1 (distal), a faixa etária $<20$ anos

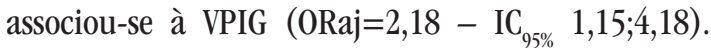
Nenhuma das variáveis do nível 2 (intermediário) apresentou significância em relação ao desfecho na análise bruta, não sendo incluídas no modelo. No modelo final, permaneceram associados com VPIG a faixa etária da mulher $<20$ anos (0Raj=2,09 $-\mathrm{IC}_{95 \%}$ $1,17 ; 3,54)$ e o consumo de drogas ilícitas pelo parceiro íntimo (0Raj=8,78 - IC $\left.\mathrm{I5 \%}_{2} 2,13 ; 28,92\right)$.

\section{Discussão}

Este estudo é o primeiro a estimar a prevalência e os fatores associados à VPIG em mulheres residentes em Caxias. 0s dados evidenciaram elevada prevalência de VPIG, sendo a ocorrência de violência psicológica isolada a mais relatada, seguida da ocorrência simultânea de violência física e psicológica. Um grupo de variáveis de potenciais fatores de risco para VPIG surgiu da análise bruta. Mulheres jovens, com histórico de violência pessoal ou familiar, com parceiro sem ocupação e/ou usuário de drogas ilícitas, apresentaram maior chance de serem vítimas de violência na gestação. Contudo, na análise hierarquizada, permaneceram associados à ocorrência de VPIG apenas o consumo de drogas ilícitas pelo parceiro e a faixa etária inferior a 20 anos.

A prevalência da VPIG observada em Caxias foi superior à encontrada na maioria de outros estudos. ${ }^{2,5,15}$ Dados da prevalência de VPIG em 19 países, entre 1998 e 2007, evidenciaram magnitude entre 2,0 e 13,5\%. ${ }^{3}$ Padrão semelhante foi observado em pesquisa realizada na cidade do Rio de Janeiro, entre 2006 e 2007, quando se constatou a prevalência da VPIG em $5,1 \%$ das entrevistadas. ${ }^{20}$ Prevalências mais elevadas, similares à encontrada na atual pesquisa, foram verificadas em inquéritos na zona rural pernambucana, entre 2000 e 2003 (32\%), ${ }^{6}$ e na capital São Luís, MA, em 2010 (49,6\%). ${ }^{21}$ É possível que a elevada prevalência de VPIG na região Nordeste esteja relacionada ao contexto social e estrutural, com normas e valores característicos de uma cultura patriarcal, considerada impulsora da violência contra a mulher.

Diversos fatores podem contribuir para as discrepâncias nas prevalências de VPIG. Em primeiro lugar, vale a pena considerar que diferentes instrumentos são utilizados para identificar esse fenômeno. Um dos pontos positivos do uso do WHO VAW seria a comparabilidade entre estudos realizados em localidades e regiões de contextos socioculturais diversos. ${ }^{7,9,11,14,15}$ Em segundo lugar, o tamanho amostral e o período gestacional das participantes dos estudos também podem influenciar o desfecho. A inclusão de mulheres em todos os períodos gestacionais, assim como a avaliação retrospectiva da VPIG, realizada no período pós-parto, pode contribuir para a diferença nas prevalências encontradas. ${ }^{12,13}$ Além disso, diferentes definições de VPIG foram usadas nas pesquisas consultadas. ${ }^{13,20}$ Entretanto, é possível que os indicadores de ocorrência VPIG não reflitam a real magnitude do problema: muitas mulheres não declaram os atos violentos de que são vítimas, seja pelo medo de relatar tais situações, seja pela dependência financeira e emocional do companheiro. ${ }^{2,18}$

Neste estudo, observou-se maior frequência de violência psicológica, seguida pela ocorrência concomitante, psicológica e física, conforme observado em outras pesquisas., ${ }^{8,12,15,21} 0$ predomínio de violência psicológica na maioria das vítimas de VPI pode estar relacionado ao constrangimento em relatar tipos mais graves de abusos conjugais e, ainda, ao medo de ser alvo de julgamentos. ${ }^{13,15}$

0 uso de drogas ilícitas pelo parceiro íntimo, verificado pela análise hierarquizada dos casos investigados em Caxias, representou fator de risco para a VPIG, assim como observado em outras localidades. ${ }^{8,12,22}$ Dados de 1.379 mulheres residentes em Campinas, SP, demonstraram que o uso de drogas ilícitas pelo parceiro quase dobrou a chance de a mulher ser vítima de violência física e sexual na gestação. ${ }^{12}$ Em Vitória, ES, o consumo de drogas pelo companheiro também aumentou as chances de mulheres serem vítimas de violência psicológica, ${ }^{23}$ tornando-as mais vulneráveis a maus-tratos conjugais.

A utilização de drogas pelo agressor, pela vítima ou por ambos relaciona-se com uma ampla gama de 
Tabela 1 - Associação de variáveis sociodemográficas e comportamentais com a ocorrência de violência por parceiro íntimo na gestação, Caxias, Maranhão, 2019-2020

\begin{tabular}{|c|c|c|c|c|c|c|c|}
\hline \multirow{2}{*}{ Variáveis } & \multicolumn{2}{|c|}{ Todos $(n=233)$} & \multicolumn{2}{|c|}{ VPIGa $(n=77)$} & \multirow{2}{*}{ ORbrb } & \multirow{2}{*}{ IC $_{95 \%}{ }^{\mathrm{C}}$} & \multirow{2}{*}{ p-valor } \\
\hline & $\mathbf{n}$ & $\%$ & $\mathbf{n}$ & $\%$ & & & \\
\hline \multicolumn{8}{|c|}{ Faixa etária (em anos) } \\
\hline$<20$ & 78 & 33,5 & 37 & 48,0 & 2,59 & 1,$47 ; 4,59$ & 0,001 \\
\hline$\geq 20$ & 155 & 66,5 & 40 & 52,0 & 1,00 & & \\
\hline \multicolumn{8}{|c|}{ Raça/cor da pele ${ }^{d}$} \\
\hline Branca & 11 & 4,7 & 2 & 2,6 & 1,00 & & \\
\hline Preta & 209 & 89,7 & 71 & 92,2 & 2,15 & 0,$43 ; 10,81$ & 0,352 \\
\hline \multicolumn{8}{|c|}{ Escolaridade (em anos de estudo) } \\
\hline$\leq 8$ & 89 & 38,2 & 34 & 44,1 & 1,00 & & \\
\hline$\geq 9$ & 144 & 61,8 & 43 & 55,9 & 0,68 & 0,$39 ; 1,20$ & 0,189 \\
\hline \multicolumn{8}{|c|}{ Situação conjugal } \\
\hline Com parceiro & 189 & 81,1 & 59 & 76,6 & 1,00 & & \\
\hline Sem parceiro & 44 & 18,9 & 18 & 23,4 & 1,52 & 0,$78 ; 2,99$ & 0,220 \\
\hline \multicolumn{8}{|c|}{ Fonte de renda própria } \\
\hline $\operatorname{Sim}$ & 64 & 27,5 & 15 & 19,5 & 1,00 & & \\
\hline Não & 169 & 72,5 & 62 & 80,5 & 1,89 & 0,$98 ; 3,65$ & 0,057 \\
\hline \multicolumn{8}{|l|}{ Ocupação } \\
\hline Com ocupação & 58 & 24,9 & 16 & 20,8 & 1,00 & & \\
\hline Sem ocupação & 175 & 75,1 & 61 & 79,2 & 1,40 & 0,$73 ; 2,70$ & 0,309 \\
\hline \multicolumn{8}{|c|}{ Condição de moradia } \\
\hline Casa própria & 168 & 72,1 & 53 & 68,8 & 1,00 & & \\
\hline Casa alugada & 65 & 27,9 & 24 & 31,2 & 1,27 & 0,$69 ; 2,31$ & 0,435 \\
\hline \multicolumn{8}{|c|}{ Consumo de álcool pela gestante } \\
\hline Sim & 62 & 26,6 & 23 & 29,9 & 1,27 & 0,$69 ; 2,35$ & 0,429 \\
\hline Não & 171 & 73,4 & 54 & 70,1 & 1,00 & & \\
\hline \multicolumn{8}{|c|}{ Consumo de drogas ilícitas pela gestante } \\
\hline $\operatorname{Sim}$ & 6 & 2,6 & 3 & 3,9 & 2,06 & 0,$41 ; 10,49$ & 0,381 \\
\hline Não & 227 & 97,4 & 74 & 96,1 & 1,00 & & \\
\hline \multicolumn{8}{|c|}{ Faixa etária do parceiroe } \\
\hline$<20$ & 32 & 13,7 & 11 & 14,3 & 0,96 & 0,$47 ; 2,28$ & 0,923 \\
\hline$\geq 20$ & 197 & 84,6 & 66 & 85,7 & 1,00 & & \\
\hline \multicolumn{8}{|c|}{ Raça/cor da pele do parceiro ${ }^{f}$} \\
\hline Branca & 50 & 21,5 & 14 & 18,2 & 1,00 & & \\
\hline Negra & 167 & 71,7 & 56 & 72,7 & 1,17 & 0,$53 ; 2,63$ & 0,696 \\
\hline \multicolumn{8}{|c|}{ Escolaridade do parceiro (em anos de estudo) ${ }^{g}$} \\
\hline$\leq 8$ & 85 & 36,5 & 32 & 41,5 & 1,00 & & \\
\hline$\geq 9$ & 127 & 54,5 & 39 & 50,6 & 0,73 & 0,$41 ; 1,31$ & 0,295 \\
\hline
\end{tabular}


Continuação

Tabela 1 - Associação de variáveis sociodemográficas e comportamentais com a ocorrência de violência por parceiro íntimo na gestação, Caxias, Maranhão, 2019-2020

\begin{tabular}{|c|c|c|c|c|c|c|c|}
\hline \multirow{2}{*}{ Variáveis } & \multicolumn{2}{|c|}{ Todos $(n=233)$} & \multicolumn{2}{|c|}{ VPIGa $(n=77)$} & \multirow{2}{*}{ ORbrb } & \multirow{2}{*}{ IC $_{95 \%}{ }^{\mathrm{C}}$} & \multirow{2}{*}{ p-valor } \\
\hline & $\mathbf{n}$ & $\%$ & $\mathbf{n}$ & $\%$ & & & \\
\hline \multicolumn{8}{|c|}{ Ocupação do parceiro } \\
\hline Com ocupação & 200 & 85,8 & 58 & 75,3 & 1,00 & & \\
\hline Sem ocupação & 33 & 14,2 & 19 & 24,7 & 3,32 & 1,$51 ; 7,02$ & 0,002 \\
\hline \multicolumn{8}{|c|}{ Consumo de álcool pelo parceiro } \\
\hline Sim & 149 & 63,9 & 54 & 70,1 & 1,50 & 0,$85 ; 2,71$ & 0,169 \\
\hline Não & 84 & 36,1 & 23 & 29,9 & 1,00 & & \\
\hline \multicolumn{8}{|c|}{ Consumo de drogas pelo parceiro } \\
\hline Sim & 17 & 7,3 & 14 & 18,2 & 11,33 & 3,$15 ; 40,80$ & $<0,001$ \\
\hline Não & 216 & 92,7 & 63 & 81,8 & 1,00 & & \\
\hline
\end{tabular}

a) VPIG: violência por parceiro íntimo na gestação; b) ORbr: odds ratio bruta; c) IC $\mathrm{C}_{95 \%}$ : intervalo de confiança de $95 \%$; d) Não sabe/não quis responder: $2(0,9 \%)$; amarela, 10 (4,3\%)/3 (1,3\%) com VPIG; indígena, 1 (0,4\%)/1 (1,3\%) com VPIG; e) Não sabe/não quis responder: 4 (1,7\%); f) Amarela: 15 (6,4\%)/3 (1,3\%) com VPIG; indígena, 1 (0,4\%)/1 (1,3\%) com VPIG; g) Não sabe/não quis responder: $21(9,0 \%) / 6(7,8 \%)$ com VPIG.

Tabela 2 - Associação de variáveis obstétricas e histórico de violência com a ocorrência de violência por parceiro íntimo na gestação, Caxias, Maranhão, 2019-2020

\begin{tabular}{|c|c|c|c|c|c|c|c|}
\hline \multirow{2}{*}{ Variáveis } & \multicolumn{2}{|c|}{ Todos $(n=233)$} & \multicolumn{2}{|c|}{ VPIGa $(n=77)$} & \multirow{2}{*}{ ORbr } & \multirow{2}{*}{$\mathrm{IC}_{95 \%} \mathrm{C}^{\mathrm{C}}$} & \multirow{2}{*}{ p-valor } \\
\hline & $\mathbf{n}$ & $\%$ & $\mathbf{n}$ & $\%$ & & & \\
\hline \multicolumn{8}{|c|}{ Violência física antes dos 15 anos } \\
\hline Sim & 131 & 56,2 & 55 & 71,4 & 2,63 & 1,$46 ; 4,73$ & 0,001 \\
\hline Não & 102 & 43,8 & 22 & 28,6 & 1,00 & & \\
\hline \multicolumn{8}{|c|}{ Violência física após os 15 anos ${ }^{\mathrm{d}}$} \\
\hline Sim & 40 & 17,2 & 20 & 26,0 & 2,45 & 1,$22 ; 4,93$ & 0,012 \\
\hline Não & 183 & 78,5 & 53 & 68,8 & 1,00 & & \\
\hline \multicolumn{8}{|c|}{ Violência sexual antes dos 15 anos } \\
\hline Sim & 15 & 6,4 & 9 & 11,7 & 3,30 & 1,$13 ; 9,67$ & 0,029 \\
\hline Não & 218 & 93,6 & 68 & 88,3 & 1,00 & & \\
\hline \multicolumn{8}{|c|}{ Violência sexual após os 15 anos ${ }^{\mathrm{d}}$} \\
\hline Sim & 18 & 7,7 & 9 & 11,7 & 2,20 & 0,$83 ; 5,81$ & 0,110 \\
\hline Não & 205 & 88,0 & 64 & 83,1 & 1,00 & & \\
\hline \multicolumn{8}{|c|}{ Violência psicológica antes dos 15 anos } \\
\hline Sim & 119 & 51,1 & 54 & 70,1 & 3,28 & 1,$84 ; 5,89$ & $<0,001$ \\
\hline Não & 114 & 48,9 & 23 & 29,9 & 1,00 & & \\
\hline \multicolumn{8}{|c|}{ Violência psicológica após os 15 anos $^{e}$} \\
\hline Sim & 100 & 42,9 & 45 & 58,4 & 2,77 & 1,$59 ; 4,94$ & 0,001 \\
\hline Não & 123 & 52,8 & 28 & 36,4 & 1,00 & & \\
\hline
\end{tabular}


Continuação

Tabela 2 - Associação de variáveis obstétricas e histórico de violência com a ocorrência de violência por parceiro íntimo na gestação, Caxias, Maranhão, 2019-2020

\begin{tabular}{|c|c|c|c|c|c|c|c|}
\hline \multirow{2}{*}{ Variáveis } & \multicolumn{2}{|c|}{ Todos $(n=233)$} & \multicolumn{2}{|c|}{ VPIGa $(n=77)$} & \multirow{2}{*}{ ORbrb } & \multirow{2}{*}{ IC $_{95 \%}{ }^{\mathrm{C}}$} & \multirow{2}{*}{ p-valor } \\
\hline & $\mathbf{n}$ & $\%$ & $\mathbf{n}$ & $\%$ & & & \\
\hline \multicolumn{8}{|c|}{ Agressão materna presenciada } \\
\hline Sim & 67 & 28,8 & 28 & 36,4 & 1,71 & 0,$95 ; 4,94$ & 0,073 \\
\hline Não & 166 & 71,2 & 49 & 63,6 & 1,00 & & \\
\hline \multicolumn{8}{|c|}{ Tipo de agressão materna presenciadae } \\
\hline Física e psicológica & 24 & 10,3 & 14 & 18,2 & 2,90 & 1,$03 ; 8,14$ & 0,043 \\
\hline Outras & 43 & 18,5 & 14 & 18,2 & 1,00 & & \\
\hline \multicolumn{8}{|c|}{ Número de gestações } \\
\hline$\leq 2$ & 157 & 67,4 & 53 & 68,9 & 1,00 & & \\
\hline$>2$ & 76 & 32,6 & 24 & 31,2 & 0,90 & 0,$50 ; 1,63$ & 0,740 \\
\hline \multicolumn{8}{|l|}{ Número de partos } \\
\hline$\leq 2$ & 203 & 87,1 & 69 & 89,6 & 1,00 & & \\
\hline$>2$ & 30 & 12,9 & 8 & 10,4 & 0,70 & 0,$29 ; 1,67$ & 0,426 \\
\hline \multicolumn{8}{|c|}{ Antecedente de aborto } \\
\hline Sim & 51 & 21,9 & 18 & 23,4 & 1,00 & & \\
\hline Não & 182 & 78,1 & 59 & 76,6 & 0,87 & 0,$46 ; 1,69$ & 0,700 \\
\hline \multicolumn{8}{|c|}{ Número de consultas de pré-natal } \\
\hline$<6$ & 114 & 48,9 & 40 & 51,9 & 1,00 & & \\
\hline$\geq 6$ & 119 & 51,1 & 37 & 48,1 & 0,83 & 0,$48 ; 1,44$ & 0,517 \\
\hline
\end{tabular}

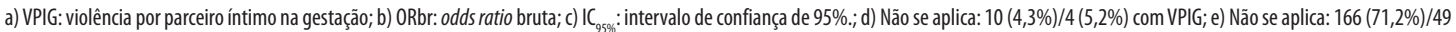
$(63,6 \%)$ com VPIG.

episódios de violência doméstica, podendo culminar com traumas físicos que colocam em risco a vida da mulher. ${ }^{24,25}$ Por reduzir a capacidade de controlar impulsos, o uso de substâncias ilícitas pelo companheiro torna a mulher mais vulnerável a conflitos e, com frequência, desencadeia um ciclo vicioso de violência. ${ }^{22}$ Discussões, culpabilização da vítima, minimização das agressões e promessas de mudança de comportamento fazem parte do roteiro habitual de VPIG quando há consumo de drogas ilícitas pelo parceiro. ${ }^{19,25,26}$

É válido destacar que, embora o uso de drogas tenha papel importante na ocorrência de episódios violentos, atuando como possível potencializador da agressão, não está totalmente elucidada sua relação com a ocorrência de violência. ${ }^{26}$ Porém, à medida que se reduz o consumo de drogas ilícitas pelo parceiro íntimo, também diminuem os casos de violência contra a mulher. ${ }^{24,26}$ Portanto, faz-se necessário o desenvolvimento de ações que articulem as questões de gênero, além das relacionadas ao consumo de drogas lícitas e ilícitas. ${ }^{23,24}$ Não obstante vários estudos demonstrarem o uso de álcool pelo parceiro e, como decorrência, a criação de condições favoráveis à violência, ${ }^{12,14,19} 0$ presente estudo não encontrou associação dessa variável com a VPIG. Fatores como tamanho amostral pequeno, tipologia variada da violência e formas diferentes de identificar o consumo de álcool influenciam essa associação. ${ }^{19}$ Também é possível que 0 resultado desta pesquisa esteja relacionado à força da associação observada com drogas ilícitas, mascarando o efeito do álcool.

Um estudo realizado em dez países, incluindo 0 Brasil, entre os anos 2000 e 2003, constatou que a idade mais jovem esteve fortemente associada ao aumento do risco de VPI, em todos esses países, ${ }^{27} \mathrm{se}-$ melhantemente aos dados deste trabalho. Pesquisas realizadas na cidade do Rio de Janeiro e em Recife, $\mathrm{PE}$, também verificaram prevalência mais elevada de VPIG em mulheres com idade inferior a 20 anos. ${ }^{7,20}$ 
Tabela 3 - Análise hierarquizada dos fatores associados à violência por parceiro íntimo na gestação, Caxias, Maranhão, 2019-2020

\begin{tabular}{|c|c|c|c|c|c|c|}
\hline \multirow{2}{*}{ Variáveis } & \multicolumn{3}{|c|}{ Modelo $1^{\text {a }}$} & \multicolumn{3}{|c|}{ Modelo $2^{b}$} \\
\hline & ORajc & $I_{95 \%}{ }^{d}$ & p-valor & ORajc & $I_{95 \%}{ }^{d}$ & p-valor \\
\hline \multicolumn{7}{|c|}{ Faixa etária (em anos) } \\
\hline$<20$ & 2,18 & 1,$15 ; 4,18$ & 0,023 & 2,09 & 1,$17 ; 3,54$ & 0,031 \\
\hline$\geq 20$ & 1,00 & & & 1,00 & & \\
\hline \multicolumn{7}{|l|}{ Fonte de renda } \\
\hline Sim & 1,00 & & & 1,00 & & \\
\hline Não & 1,08 & 0,$86 ; 2,56$ & 0,211 & 1,06 & 0,$84 ; 2,45$ & 0,465 \\
\hline \multicolumn{7}{|c|}{ Ocupação profissional do parceiro } \\
\hline Sim & 1,00 & & & 1,00 & & \\
\hline Não & 1,87 & 0,$79 ; 4,33$ & 0,372 & 1,51 & 0,$82 ; 3,65$ & 0,127 \\
\hline \multicolumn{7}{|c|}{ Consumo de drogas pelo parceiro } \\
\hline Sim & & & & 8,78 & 2,$13 ; 28,92$ & 0,001 \\
\hline Não & & & & 1,00 & & \\
\hline \multicolumn{7}{|c|}{ Violência física antes dos 15 anos } \\
\hline Sim & & & & 1,55 & 0,$93 ; 4,55$ & 0,134 \\
\hline Não & & & & 1,00 & & \\
\hline \multicolumn{7}{|c|}{ Violência física após os 15 anos } \\
\hline Sim & & & & 1,73 & 0,$81 ; 3,60$ & 0,342 \\
\hline Não & & & & 1,00 & & \\
\hline \multicolumn{7}{|c|}{ Violência sexual antes dos 15 anos } \\
\hline Sim & & & & 1,44 & 0,$67 ; 4,59$ & 0,320 \\
\hline Não & & & & 1,00 & & \\
\hline \multicolumn{7}{|c|}{ Violência psicológica antes dos 15 anos } \\
\hline Sim & & & & 1,68 & 0,$75 ; 6,01$ & 0,128 \\
\hline Não & & & & 1,00 & & \\
\hline \multicolumn{7}{|c|}{ Violência psicológica após os 15 anos } \\
\hline Sim & & & & 1,33 & 0,$56 ; 5,74$ & 0,530 \\
\hline Não & & & & 1,00 & & \\
\hline \multicolumn{7}{|c|}{ Agressão materna presenciada } \\
\hline Sim & & & & 1,23 & 0,$82 ; 2,96$ & 0,125 \\
\hline Não & & & & 1,00 & & \\
\hline \multicolumn{7}{|c|}{ Tipo de agressão materna presenciada } \\
\hline Física e psicológica & & & & 1,94 & 0,$74 ; 6,04$ & 0,076 \\
\hline Outras & & & & 1,00 & & \\
\hline
\end{tabular}

a) Nível 1 (distal) ajustado isoladamente; b) Nível 3 (proximal) ajustado pelas variáveis do nível 1; c) ORaj: odds ratio ajustada; d) IC ; : intervalo de confiança de $95 \%$. 
No entanto, em São Luís, MA, ${ }^{21}$ e na cidade de São Pau$10,{ }^{13} \mathrm{a}$ VPIG foi mais presente em mulheres com 25 anos de idade ou mais, enquanto na Turquia, a faixa etária dos 45 aos 49 anos foi apontada como fator de risco para a VPI entre mulheres grávidas. ${ }^{26}$ Não há consenso sobre a idade jovem da mulher constituir fator isolado para a ocorrência de VPI; esta pode se associar a outras condições determinantes, como baixa escolaridade, desemprego, dependência financeira do parceiro íntimo, e gravidez precoce e não planejada., ${ }^{2,913}$ Dessa forma, 0 aumento da idade, do nível de escolaridade e a maior independência financeira revelam-se características protetoras, associadas a menor exposição e tolerância da mulher à violência do parceiro. ${ }^{19,25}$

As características sociodemográficas e comportamentais da mulher e do parceiro íntimo, assim como a exposição da mulher à violência em períodos que antecederam a gestação, têm sido apontadas como fatores de risco para a VPIG., ${ }^{2,7,13,20,21,27,28}$ Os dados deste estudo vão ao encontro dos principais achados da literatura. A análise hierarquizada demonstrou que a ausência de fonte de renda própria e o histórico de violência da mulher não representaram fatores de risco para a ocorrência da VPIG. Da mesma maneira, a ausência de ocupação profissional do parceiro íntimo também se associou à violência na gestação.

Embora seja um conhecido fator de risco para a VPI, ${ }^{2,19,22,28}$ a parceria íntima sem ocupação não se associou à VPIG, segundo os resultados da investigação em Caxias. No município de Maringá, PR, ao contrário, o desemprego do parceiro íntimo apresentou associação significativa com a VPIG, ${ }^{22}$ e também com a violência física contra a mulher gestante em Recife. ${ }^{28}$ Todavia não foi esclarecida a razão pela qual o desemprego do parceiro aumenta as chances de exposição à VPI, inclusive no período gestacional. É possível que haja uma inter-relação dessa violência com fatores associados ao desemprego, como a pobreza e a incapacidade de o parceiro cumprir o papel de provedor, especialmente em um contexto de aumento da família por conta da gravidez., ${ }^{2,29}$

Divergindo dos achados encontrados com frequência, em estudos nacionais e internacionais, $, 925,29$ tanto a exposição à violência física e psicológica, antes e depois dos 15 anos, como a ocorrência de violência sexual anterior a essa idade, não permaneceram associadas ao desfecho na análise hierarquizada do presente estudo.
Segundo dados de uma pesquisa conduzida em 2014, na cidade de Florianópolis, SC, mulheres que sofreram violência do parceiro nos 12 meses anteriores à gestação apresentaram ${ }^{15,8}$ mais chances de serem expostas a essa violência. ${ }^{9}$ No entanto, a VPIG não costuma ser um evento isolado e terminal: estima-se que 60 a $96 \%$ das vítimas de VPIG foram expostas antes do período gestacional, e que os episódios na gestação sejam apenas a continuidade de eventos pregressos. ${ }^{29}$ Cumpre também acrescentar que ter presenciado agressões maternas mostrou-se um fator associado à VPIG: mulheres que viram suas mães sofrerem VPI apresentam até três vezes mais chances de serem vítimas de VPIG, ${ }^{28}$ havendo, ainda, o risco de seu testemunho reforçar uma percepção equivocada dessa forma de violência e sua normalização como um fato inerente às relações conjugais, e consequentemente, uma tolerância maior à VPI, muito embora inadimissível. ${ }^{16,26}$

0 presente estudo apresenta limitações a serem consideradas. Em primeiro lugar, o desenho transversal do estudo não permite estabelecer relação de temporalidade entre a VPIG e as variáveis analisadas. Em segundo lugar, a abordagem não incluiu mulheres entre o primeiro e o segundo trimestres de gestação, apesar de, nesse período inicial da gravidez, não estarem isentas de atitudes e ações violentas vindas de um parceiro íntimo. Da mesma maneira, os resultados da investigação dizem respeito apenas às mulheres que realizaram o acompanhamento pré-natal nos serviços públicos de saúde de Caxias, desconhecendo-se a prevalência e os fatores associados à VPIG de gestantes acompanhadas em serviços privados do município. Em terceiro e último lugar, as participantes não foram selecionadas por amostragem probabilística, sendo possível que a amostra pesquisada não represente, de forma fidedigna, o universo investigado.

A despeito das limitações consideradas, o estudo merece destaque pelo fato de ser o primeiro a avaliar a VPIG em Caxias, onde se observa frequente ocorrência de episódios de violência contra as gestantes e, como conclusão, sua insegurança e risco provocados por essa violência durante a gravidez. 0 s resultados encontrados podem fornecer subsídios para o planejamento de estratégias e a implementação de ações em resposta à violência contra a mulher gestante, provocada por parceiro íntimo, no município de Caxias. Os serviços de atenção primária em saúde desempenham papel 
significativo na identificação da agressão sofrida pela mulher grávida. Todo profissional de saúde envolvido no cuidado pré-natal deve estar atento, tanto aos indícios da presença quanto às oportunidades de revelação dessa forma de violência, e oferecer seu apoio a essas mulheres não apenas quando solicitado. Estratégias como a escuta ativa, a abstenção de julgamento, o conforto moral e a informação dos direitos legais envolvidos são mais do que importantes para uma decisão resolutiva da mulher, diante da violência do parceiro sobre seu corpo e seu concepto.

\section{Referências}

1. Kusuma YS, Babu BV. Elimination of violence against women and girls as a global action agenda. J Inj Violence Res [Internet]. 2017 Jul [cited 2020 0ct 10];9(2):117-21. Available from: https://doi.org/10.5249/jivr.v9i2.908

2. Coll CVN, Ewerling F, García-Moreno C, Hellwig F, Barros AJD. Intimate partner violence in 46 low-income and middle-income countries: an appraisal of the most vulnerable groups of women using national health surveys. BMJ Global Health [Internet]. 2020 Jan [cited 2020 0ct 10];5(1):e002208. Available from: https://doi.org/0.1136/bmjgh-2019-002208

3. Jewkes RK, Dunkle K, Nduna M, Shai N. Intimate partner violence, relationship power inequity, and incidence of HIV infection in young women in South Africa: a cohort study. Lancet [Internet]. $2010 \mathrm{Jul}$ [cited 2020 May 12];376(9734):41-8. Available from: https://doi.org/10.1016/S0140-6736(10)60548-X

4. Devries KM, Mak JY, Bacchus LJ, Child JC, Falder $\mathrm{G}$, Petzold $\mathrm{M}$, et al. Intimate partner violence and incident depressive symptoms and suicide attempts: a systematic review of longitudinal studies. PLoS Med [Internet]. 2013 May [cited 2020 Apr 17];10(5):e1001439. Available from: https://doi.org/10.1371/journal.pmed.1001439

5. Devries KM, Kishor S, Johnson H, Stöckl H, Backus LJ, Garcia-Moreno C, et al. Intimate partner violence during pregnancy: analysis of prevalence data from 19 countries. Reprod Health Matters [Internet]. 2010 Nov [cited 2020 Apr 17];18(36):158-70. Available from: https://doi.org/10.1016/S0968-8080(10)36533-5

6. Garcia-Moreno C, Jansen HA, Ellsberg M, Heise L, Watts $\mathrm{CH}$. Prevalence of intimate partner violence: findings from the WHO multi-country study on women's health

\section{Contribuição dos autores}

Conceição HN e Madeiro AP contribuíram na concepção e delineamento do estudo, análise e interpretação dos resultados, redação e revisão crítica do conteúdo do manuscrito. Coelho SF contribuiu na interpretação dos dados, redação e revisão crítica do conteúdo do manuscrito. Os três autores aprovaram a versão final do manuscrito e são responsáveis por todos os seus os aspectos, incluindo a garantia de sua precisão e integridade.

and domestic violence. Lancet [Internet]. 2006 0ct [cited 2020 Jun 22];368(9543):1260-9. Available from: https://doi.org/10.1016/S0140-6736(06)69523-8

7. Carneiro JF, Valongueiro S, Ludermir AB, Araújo TVB. Violência física pelo parceiro íntimo e uso inadequado do pré-natal entre mulheres do Nordeste do Brasil. Rev Bras Epidemiol [Internet]. 2016 abr-jun [citado 2019 maio 3];19(2):243-55. Disponível em: https://doi.org/10.1590/1980-5497201600020003

8. Viellas EF, Gama SGN, Carvalho ML, Pinto LW. Fatores associados à agressão física em gestantes e os desfechos negativos no recém-nascido. J Pediatr [Internet]. 2013 jan-fev [citado 2019 jul 25];89(1):83-90. Disponível em: https://doi.org/10.1016/j.jped.2013.02.013

9. Pires MRM, Locatelli TZ, Rojas PFB, Lindner SR, Bolsoni CC, Coelho EBS. Prevalência e os fatores associados da violência psicológica contra gestantes em capital no Sul do Brasil. Saúde Transf Soc [Internet]. 2017 [citado 2020 jun 22];8(1):29-39. Disponível em: http://incubadora.periodicos.ufsc.br/ index.php/saudeetransformacao/article/view/3797

10. Chisholm CA, Bullock L, Ferguson II JE. Intimate partner violence and pregnancy: epidemiology and impact. Am J Obstet Gynecol [Internet]. 2017 Aug [cited 2020 Jan 2];217(2):141-4. Available from: https://doi.org/10.1016/j.ajog.2017.05.042

11. Costa DCS, Ribeiro MRC, Batista RFL, Valente CM, Ribeiro JVF, Almeida LA, et al. Factors associated with physical violence against pregnant women from São Luís, Maranhão State, Brazil: an approach using structural equation modeling. Cad Saúde Pública [Internet]. 2017 Jan [cited 2020 Jan 2];33(1):e00078515. Available from: https://doi.org/10.1590/0102-311x00078515 
12. Audi CAF, Segall-Corrêa AM, Santiago SM, Andrade MGG, Pérez-Escamilla R. Violência doméstica na gravidez: prevalência e fatores associados. Rev Saúde Pública [Internet]. 2008 out [citado 2020 jun 22];42(5):877-85. Disponível em: https:// doi.org/10.1590/S0034-89102008005000041

13. Durand JG, Schraiber LB. Violência na gestação entre usuárias de serviços públicos de saúde da Grande São Paulo: prevalência e fatores associados. Rev Brasil Epidemiol [Internet]. 2007 set [citado 2019 jul 25];10(3):310-22. Disponível em: https:// doi.org/10.1590/S1415-790X2007000300003

14. Mahenge B, Stöckl H, Abubakari A, Mbwambo J, Jahn A. Physical, sexual, emotional and economic intimate partner violence and controlling behaviors during pregnancy and postpartum among women in Dar es Salaam, Tanzania. PLoS One [Internet]. 2016 Oct [cited 2020 Jun 22];11(10):e0164376. Available from: https://doi.org/10.1371/journal.pone.0164376

15. Barros EN, Silva MA, Falbo-Neto GH, Lucena SG, Ponzo L, Pimentel AP. Prevalência e fatores associados à violência por parceiro íntimo em mulheres de uma comunidade em Recife/Pernambuco, Brasil. Ciênc Saúde Coletiva [Internet]. 2016 fev [citado 2019 abr 13];21(2):591-8. Disponível em: https:// doi.org/10.1590/1413-81232015212.10672015

16. Instituto Brasileiro de Geografia e Estatística -IBGE. Caxias. Panorama [Internet]. Rio de Janeiro: IBGE; 2020 [citado 2020 set 20]. Disponível em: https:// cidades.ibge.gov.br/brasil/ma/caxias/panorama

17. Schraiber LB, Latorre MRD, França Jr I, Segri NJ, D'Oliveira AFPL. Validade do instrumento WHO VAW STUDY para estimar violência de gênero contra a mulher. Rev Saúde Pública [Internet]. 2010 ago [citado 2019 jul 25];44(4):658-66. Disponível em: https://doi.org/10.1590/S0034-89102010000400009

18. World Health Organization - WHO. Global and regional estimates of violence against women: prevalence and health effects of intimate partner violence and non-partner sexual violence [Internet]. Geneva: WHO; 2013 [cited 2020 May 22]. Available from: https://www.who.int/reproductivehealth/ publications/violence/9789241564625/en/

19. Silva EP, Valongueiro S, Araújo TVB, Ludermir $A B$. Incidência e fatores de risco para violência por parceiro íntimo no período pós-parto. Rev Saúde Pública [Internet]. 2015 ago [citado 2019 abr 13];49(5):46-55. Disponível em: https://doi. org/10.1590/S0034-8910.2015049005432
20. Santos SA, Lovisi GM, Valente CDCB, Legay L, Abelha L. Violência doméstica durante a gestação: um estudo descritivo em uma unidade básica de saúde no Rio de Janeiro. Cad Saúde Coletiva [Internet]. 2010 out-dez [citado 2020 jan 2];18(4):483-93. Disponível em: http:/www.cadernos.iesc.ufrj.br/cadernos/images/ csc/2010_4/artigos/CSC_v18n4_483-493.pdf

21. Aguiar LC. Violência na gravidez: caracterização de casos em São Luís (MA) no ano de 2010 [dissertação]. São Luís (MA): Universidade Federal do Maranhão; 2014. Disponível em: https://renasf.fiocruz.br/ sites/renasf.fiocruz.br/files/disseracoes/2014_ UFMA_Lia\%20Cardoso\%20de\%20Aguiar.pdf

22. Sgobero JKGS, Monteschio LVC, Zurita RCM, Oliveira RR, Freitas-Mathias TA. Violência física por parceiro íntimo na gestação: prevalência e alguns fatores associados. Aquichan [Internet]. 2015 set [citado 2020 jul 12];15(3):339-50. Disponível em: https://doi.org/10.5294/aqui.2015.15.3.3

23. Leite FMC, Luis MA, Amorim MHC, Maciel ELN, Gigante DP. Violência contra a mulher e sua associação com o perfil do parceiro íntimo: estudo com usuárias da atenção primária. Rev Brasil Epidemiol [Internet]. 2019 [citado 2020 maio 3];22: e190056. Disponível em: https://doi.org/10.1590/1980-549720190056

24. Vieira LB, Cortes LF, Padoin SMM, Souza IEO, Paula CC, Terra MG. Abuso de álcool e drogas e violência contra as mulheres: denúncias de vividos. Rev Bras Enferm [Internet]. 2014 [citado 2020 maio 3];67(3):366-72. Disponível em: http:// dx.doi.org/10.5935/0034-7167.20140048

25. Zilberman ML, Blume SB. Violência doméstica, abuso de álcool e substâncias psicoativas. Rev Bras Psiquiatr [Internet]. 2005 [citado 2020 jun 12];27(supl 2):51-5. Disponível em: https:// www.scielo.br/pdf/rbp/v27s2/pt_a04v27s2

26. Yüksel-Kaptanoğlu İ, Adali T. Intimate partner violence during pregnancy in Turkey: determinants from nationwide surveys. J Interpers Violence [Internet]. 2019 Mar [cited 2020 May 22];1-30. Available from: https://doi.org/10.1177/0886260519837652

27. Abramsky T, Watts CH, Garcia-Moreno C, Devries K, Kiss L, Ellsberg M, et al. What factors are associated with recent intimate partner violence? Findings from the WHO multi-country study on women's health and domestic violence. BMC Public Health [Internet]. 2011 Feb [cited 2020 May 5];11(109):1-17. Available from: https://doi.org/10.1186/1471-2458-11-109 
28. Menezes TC, Amorim MMRD, Santos LC, Faúndes A. Violência física doméstica e gestação: resultados de um inquérito no puerpério. Rev Bras Ginecol Obstet [Internet]. 2003 jun [citado 2020 maio 5];25(5):309-16. Disponível em: https://doi. org/10.1590/S0100-72032003000500002
29. Taillieu TL, Brownridge DA. Violence against pregnant women: prevalence, patterns, risk factors, theories, and directions for future research. Aggress Violent Behav [Internet]. 2010 Feb [cited 2020 Jul 12];15(1):14-35. Available from: https://doi.org/10.1016/j.avb.2009.07.013

\section{Abstract}

objective:To analyzeprevalence and factors associated with intimate partner violence during pregnancy. Methods: This was a cross-sectional study, with data obtained through interviews conducted with pregnant women aged 10 to 49 years during the third trimester of pregnancy, living in Caxias, state of Maranhão, Brazil (2019-2020). The instrument of the World Health Organization Violence Against Women Study was used to identify violence. A bierarchical analysis was performed using multiple logistic regression. Results: 233 pregnant women were interviewed. The prevalence of violence during pregnancy was $33.0 \%$, with predominance of psychological violence (18.9\%). In the final hierarchical model, women aged $<20$ years old (ORadj $=2.09-95 \%$ CI 1.17;3.54) and illicit drug use by intimate partner (ORadj $=8.78-95 \%$ CI 2.13;28.92) remained as factors associated with the outcome. Conclusion: Prevalence of violence during pregnancy was high, with illegal drug use by young women and their partners being factors associated with its occurrence.

Keywords: Violence Against Women; Intimate Partner Violence; Pregnancy; Spouse Abuse; Cross-Sectional Studies.

\section{Resumen}

Objetivo: Analizar la prevalencia y los factores asociados de la violencia de pareja durante el embarazo. Métodos: Estudio transversal, con datos obtenidos mediante entrevistas a mujeres embarazadas de 10 a 49 años en el tercer trimestre de gestación, residentes en Caxias, Maranhão, Brasil (2019-2020). Para identificar la violencia se utilizó el instrumento del Estudio sobre la Violencia contra la Mujer de la Organización Mundial de la Salud. Se realizó análisis jerárquico mediante mediante regresión logística múltiple. Resultados: Se entrevistaron 233 mujeres. La violencia durante la gestación tuvo prevalencia de 33,0\%, con predominio de violencia psicológica (18,9\%). En el modelo jerárquico final, la edad de la mujer <20 años (ORaj=2,09 $\left.I C_{95 \%}, 1,17 ; 3,54\right)$ y el consumo de drogas ilícitas por la pareja (ORaj=8,78 - IC $\left.{ }_{95 \%} 2,13 ; 28,92\right)$ se mantuvieron asociadas al desenlace violento. Conclusión: La violencia durante el embarazo tuvo alta prevalencia, siendo la baja edad de la mujer y el consumo de sustancias ilegales por la pareja, factores asociados con su ocurrencia.

Palabras clave: Violencia contra la Mujer; Violencia de Pareja; Embarazo; Maltrato Conyugal; Estudios Transversales.

Recebido em 21/09/2020

Aprovado em 18/11/2020

Editora associada: Bárbara Reis-Santos - (0) orcid.org/0000-0001-6952-0352 Editora científica: Taís Freire Galvão - @ o orcid.org/0000-0003-2072-4834 Editora geral: Leila Posenato Garcia - (1) orcid.org/0000-0003-1146-2641 